\title{
Reviews
}

\section{An Industrial Reference Fluid for Moderately High Viscosity}

\author{
Fernando J. P. Caetano, ${ }^{\dagger,}$ João M. N. A. Fareleira ${ }^{\dagger}{ }^{\dagger}$ Andreas P. Fröba, ${ }^{\S}$ Kenneth R. Harris, ${ }^{\prime \prime}$ Alfred Leipertz, ${ }^{\S}$ \\ Carla M. B. P. Oliveira, ${ }^{\dagger}$, J. P. Martin Trusler ${ }^{\perp}$ and William A. Wakeham*,\#
}

Centro de Química Estrutural, Instituto Superior Técnico, Universidade Técnica de Lisboa, Av. Rovisco Pais, 1049-001 Lisbon, Portugal, Universidade Aberta, R. da Escola Politécnica, 147, 1269-001 Lisbon, Portugal, Lehrstuhl für Technische Thermodynamik (LTT), Friedrich-Alexander-Universität Erlangen-Nürnberg, Am Weichselgarten 8, D-91058 Erlangen, Germany, School of Physical, Environmental and Mathematical Sciences, University College, University of New South Wales, Australian Defence Force Academy, Canberra, ACT 2600, Australia, Department of Chemical Engineering and Chemical Technology, Imperial College, London, SW7 2BY, United Kingdom, and University of Southampton, Highfield, Southampton, SO17 1BJ, United Kingdom

\begin{abstract}
In industrial practice, there is a demand for a reference standard for viscosity that is established for a readily available fluid to simplify the calibration of industrial viscometers for moderately high viscosities [ 50 to 125) $\mathrm{mPa} \cdot \mathrm{s}$. Diisodecyl phthalate (DIDP) has been suggested as that reference fluid, and a number of studies of its properties have been carried out in several laboratories throughout the world, within the auspices of a project coordinated by the International Association for Transport Properties. That project has now progressed to the point where it is possible to collate the results of studies of the viscosity of the fluid by a number of different techniques, so as to lead to a proposed standard reference value which will be included in the paper. To support this recommended value, the various measurements conducted have been critically reviewed, and the sample purity and other factors affecting the viscosity have been studied. Density and surface tension measurements have also been performed. This paper does not describe the individual viscosity determinations carried out in independent laboratories because these are the subject of individual publications, but it does describe the ancillary studies conducted and their relevance to the viscosity standard. In addition, the paper contains recommended values for the viscosity of liquid DIDP. The samples of DIDP to which the recommended values refer are isomeric mixtures available commercially from certain suppliers, with a minimum purity by gas chromatography of $99.8 \%$. The recommended values result from a critical examination of all the measurements conducted to date and are supported by careful arguments dealing with the likely effects of the isomeric content of the sample as well as of other impurities. The proposed reference standard is intended particularly to serve an industrial need for a readily available calibration material with a viscosity close to that required in practical situations. To that end, the recommended value has an overall relative uncertainty of approximately $1 \%$. It is therefore not intended to supersede for the reference value for the viscosity of water at $20^{\circ} \mathrm{C}$, which is known much more accurately, but rather to complement it.
\end{abstract}

\section{Introduction}

The present paper proposes an industrial reference material for viscosity, diisodecyl phthalate (DIDP), with a value of about $125 \mathrm{mPa} \cdot \mathrm{s}$ at a temperature of $293.15 \mathrm{~K}$. Following the nomenclature put forward by Marsh, ${ }^{1}$ our present endeavor concerns a "working reference material"-as it is intended mainly to "calibrate instruments" and to "check the performance of instruments"-which is also characterized as a "secondary reference material" because its value is mainly "fixed by comparison with the primary reference material" for viscosity.

According to Marsh, ${ }^{1}$ one difficulty concerning the establishment of standard reference values for viscosity has to do with

\footnotetext{
* Corresponding author. Telephone: +442380592801 . Fax: +442380593159 .

E-mail address: vice-chancellor@ soton.ac.uk.

†niversidade Técnica de Lisboa.

$\doteqdot$ Universidade Aberta.

${ }^{\S}$ Friedrich-Alexander-Universität Erlangen-Nürnberg.

"University of New South Wales.

${ }^{\perp}$ Imperial College.

\# University of Southampton.
}

the validity of the accepted standard value for the viscosity of water at $20{ }^{\circ} \mathrm{C}$. ISO in 1998 has reviewed ${ }^{2}$ this subject and recommended the value $1.0016 \mathrm{mPa} \cdot \mathrm{s}$ for the standard value and proposed a reduction of the previous uncertainty limit to $\pm 0.17 \%$. Nevertheless, Marsh suggests that there exists the possibility that the uncertainty might be as high as $0.3 \%$. ${ }^{1}$ It should be pointed out that this uncertainty is of no special consequence, as long as all the results to be compared are obtained relative to the same value of the water reference. However, if the "true" value of viscosity is required, one should be prepared to add an uncertainty of up to $\pm 0.3 \%$ to the uncertainty of viscosity measurements owing to the water reference point. ${ }^{1}$ This will become of greater importance when experimental methods, such as vibrating-wire and surface light scattering techniques, can be operated on a rigorously absolute basis.

A special problem concerning the use of reference materials for viscosity stems, primarily, from the fact that the viscosity of fluids varies over several orders of magnitude, when we 
consider fluids of economic importance in industrial and commercial activities. One practical instrument is simply never suitable for the measurement of viscosity over a wide range of the properties. As a consequence, one single reference fluid is not suitable for calibrating all of the instruments necessary to cover the whole range of viscosity for fluids of economic interest. In practice, this means either that a large range of standard liquids need to be available or that one accepts calibration with a fluid whose viscosity is several orders of magnitude different from any primary reference point for viscosity.

1.1. Calibration of Viscometers. The calibration of viscometers is usually made, directly or indirectly, using certified reference fluids, available from national metrological laboratories or from producers whose certified fluids have viscosities traceable to standards from national metrological laboratories. The certified reference fluids are usually mixtures of oils, whose composition is proprietary and for which only general descriptions are available. ${ }^{3}$ Pure fluids are not usually selected because it is argued that their viscosity can depend significantly on their purity, in a way that is not easy to predict. ${ }^{1,4}$ Certified reference fluids are supposed to be characterized by low hygroscopicity and good stability over long periods of time. ${ }^{1}$ It should, however, be noticed that Künzel et al. ${ }^{4}$ state that the utilization of most certified reference fluids should take place within strictly defined time limits.

The viscosities of the certified reference fluids must themselves be determined. This is carried out ${ }^{1}$ using a series of standard capillary viscometers in overlapping ranges, starting with a Master capillary calibrated with water at $20{ }^{\circ} \mathrm{C}$. This primary reference value is henceforth transferred, in a steppingup procedure, through a series of capillaries to one appropriate for the measurement of the viscosity of the particular certified reference fluid. ${ }^{1}$ As a consequence of this process, the uncertainty of the determination of the viscosity of the certified reference fluid builds up with the number of transfers. Künzel et al. ${ }^{4}$ report an increase in the uncertainty of the value of viscosity as the difference from the water reference point increases, stating an additional uncertainty of $\pm 0.6 \%$ on viscosity values, for viscosity up to $100 \mathrm{~Pa} \cdot \mathrm{s}$, and an uncertainty of $\pm 0.2 \%$, for viscosity smaller than $1 \mathrm{~Pa} \cdot \mathrm{s}$, if the measurement conditions are "an optimum". These and other recent estimates ${ }^{1,5}$ seem to be based on the work by Daborn, ${ }^{6}$ concerning the stability of the viscosity scale of the National Physical Laboratory (NPL), from 1952 to 1975. In our view, this restricts those estimates to excellent experimental operating conditions.

It is also noteworthy that, as remarked by Daborn, the influence of undetected systematic errors in the operation of capillary viscometers can only be assessed with resort to comparisons with independent experimental techniques. ${ }^{6}$ Naturally, the corresponding uncertainties were taken into account in the above estimates. It is noteworthy that there are some systematic errors that are very difficult to take into account in capillary viscometry. One such error arises from the differences between the surface tension of the fluids to be measured and the fluids used for calibration. ${ }^{7-9}$ The corresponding uncertainty is barely amenable to a rigorous theoretical calculation, but empirical approaches have been attempted. ${ }^{7,8}$ Another example of such a kind of error arises from the presence of residual liquid on the wall of the viscometers, ${ }^{10}$ which cannot be accounted for on a sound theoretical basis. The surface tension effects on capillary measurements are of particular concern regarding the first transfer of the step-up calibrations because it necessarily involves the use of water, which has a surface tension significantly different from that of the other fluids commonly used for the transfer. Related to this issue, it should be noted that the special design of master capillaries, namely, their increased length when compared to routine capillaries, is stated to enable a reduction of the kinetic energy and surface tension corrections. ${ }^{4}$ It is, therefore, reasonable to expect that the above estimates constitute a lower limit of the uncertainty of the stepup calibration procedures. Although some doubts may remain concerning the quantification of these effects, it seems unquestionable that the step-up calibration of capillary viscometers is responsible for the propagation of measurement uncertainties and for significant added costs.

1.2. Calibration Procedures for Routine or Practical Capillaries. The calibration of routine or practical viscometers is made either using standard reference liquids ${ }^{6}$ or by comparison with master (or reference) viscometers, the constants of which have been determined using the step-up procedure described above. Either procedure allows direct traceability to national metrological institutions. This transfer of calibration data from the realm of metrological institutions and master capillaries to nonmetrological entities and routine or practical instruments is certainly responsible for a significant increase in the uncertainty of measurement. The present authors consider that the uncertainty of the calibration constants calculated by these methods is of the order of, but presumably not less than, $\pm 1 \%$.

As an alternative, the direct calibration of routine capillaries with an industrial reference fluid (like DIDP) of an appropriate viscosity with an uncertainty of the order of $\pm 1 \%$ would yield an uncertainty for the capillary calibration that would be commensurate with the one obtained by the procedures referred to above, for a significantly lower cost. One further advantage of the use of an industrial reference fluid when compared to the current procedures lies in the fact that a reference fluid is not a proprietary substance, and its properties are available in the literature. Of particular interest for the calibration of viscometers are, beyond viscosity and density, its surface tension. This is because surface tension effects on capillary measurements can be accounted for, albeit using empirical approaches, provided that the surface tension of the calibrating fluid is known. ${ }^{7-9}$

The proposed reference standard is intended particularly to serve an industrial need for a readily available calibration material with a viscosity close to that required in practical situations. To that end, the recommended viscosity value aimed for the present standard has an overall estimated relative uncertainty of the order of $\pm 1 \%$. It is therefore not intended to substitute for the reference value for the viscosity of water at $20^{\circ} \mathrm{C}$, which is known much more accurately, but rather to complement it.

\section{Characterization of the DIDP Samples Used in the Present Work}

According to the European Union Risk Assessment Report EUR 20785, DIDP is prepared from propylene and butenes through an oligomerization process forming hydrocarbons with 8 to 15 carbon atoms. ${ }^{11}$ According to the same reference, after distillation, with the intent of obtaining nonene, aldehydes with one more carbon atom are formed by oxonation. The distribution of the $\mathrm{C}=\mathrm{C}$ double bond in the alkene and the varying effectiveness of certain catalysts determine that the position of the added carbon atom can vary, and an isomer distribution is generally created in the oxonation reaction. ${ }^{11}$ The nature of this distribution depends on the reaction conditions. $\mathrm{C} 10$ monohydric 
Table 1. Measurements of the Density of DIDP with Purity Greater than $99.8 \%$ (GC), at Atmospheric Pressure ${ }^{a, b}$

\begin{tabular}{|c|c|c|c|c|c|c|c|}
\hline method & origin \& purity & $T / \mathrm{K}$ & $N_{\mathrm{P}}$ & $\mathrm{rmsd} / \%$ & bias $/ \%$ & author & ref \\
\hline $\begin{array}{l}\text { vib. tube Anton } \\
\text { Paar DMA } 5000\end{array}$ & Merck KGaA, $99.8 \%$ & (281.15 to 315.15 ) & 18 & 0.001 & 0.000 & Caetano etal. & 15,26 \\
\hline idem & idem, & ( 273.15 to 363.15 ) & 7 & 0.004 & -0.004 & Harrisand & 16 \\
\hline idem & idem, & (283.15 to 313.15 ) & 7 & 0.003 & 0.003 & FröbaandLeipertz & 23 \\
\hline idem & idem, & ( 278.15 to 323.15 ) & 2 & 0.010 & -0.010 & Bauer & 25 \\
\hline idem & Fluka, $99.8 \%$ & (287.15 to 303.15$)$ & 18 & 0.007 & 0.007 & Caetano etal. & 26 \\
\hline pycnometer & Merck KGaA, $99.8 \%$ & (273.97 to 368.38 ) & 4 & 0.004 & -0.001 & Peletiesand Trusler & 28,29 \\
\hline
\end{tabular}

${ }^{a} N_{\mathrm{p}}$ is the number of experimental points in the temperature range $(281.15$ to 315.15$) \mathrm{K}$, used in the correlation eq $1 .{ }^{b}$ Note: The density data points used to obtain the correlation eq 1 , measured by Caetano et al., ${ }^{15,26}$ Fröba and Leipertz, ${ }^{30}$ Bauer, ${ }^{25}$ and Peleties and Trusler ${ }^{28,29}$ are available as Supporting Information to the present work.

alcohols are subsequently formed through hydrogenation of the aldehydes. ${ }^{11}$ After distillation of the resulting mixture, DIDP is formed by addition of phthalic anhydride through an esterification process. ${ }^{11,12}$

As a consequence of this production process, samples of DIDP, such as those used in the present work, are isomeric mixtures, as has been reported before ${ }^{11,13,14}$ and has been verified by some of the present authors. ${ }^{15,16}$ The qualitative studies conducted both in Portugal ${ }^{15,17}$ and Australia ${ }^{16}$ entailed ${ }^{13} \mathrm{C}$ NMR and GC-MS analysis. In the latter case, a description of the equipment used in the study has been made in ref 16 . In the former analysis, ${ }^{17}$ mass spectra were recorded on a VG TRIO-2000 Fisons instrument, operating in GC-MS mode, connected to a Carlo Erba Instruments MFC500 gas chromatographer, using a DB5-MS column (30 m length and $0.25 \mathrm{~mm}$ internal diameter) and electron impact ionization at $70 \mathrm{eV}$. Elution of the samples was made with helium at $140 \mathrm{kPa}$ as the carrier gas, and the initial temperature on the column was kept at $100{ }^{\circ} \mathrm{C}$ for $1 \mathrm{~min}$, followed by heating to $270{ }^{\circ} \mathrm{C}$ at a rate of $10{ }^{\circ} \mathrm{C} \cdot \mathrm{min}^{-1}$. The ${ }^{13} \mathrm{C} \mathrm{NMR}$ spectra were recorded ${ }^{17}$ on a Varian Unity 300 instrument, operating at $75.43 \mathrm{MHz}$ (the samples were dissolved in $\mathrm{CDCl}_{3}$, and chemical shifts were referenced to the solvent carbon signal at $77.0 \mathrm{ppm})$. The analyzed samples appear to consist of a mixture of bis(decyl) phthalates, with various types of branching along the alkyl chains. Assessment of the relative amount of all of the structures from the available data is not possible. ${ }^{17}$ Moreover, current gas chromatography (GC) analysis has not been capable of identifying the various $\mathrm{C}-10$ isomers of DIDP samples. A comparative study of the $99.8 \%$ (GC) purity samples from Merck KGaA and from Fluka (according to the respective certificates of analysis ${ }^{18,19}$ ) has shown that both samples appear to be mixtures of bis(decyl) phthalates, although the relative amounts of the components may differ between the two suppliers. The observations appear to be consistent with a different degree of branching among the two samples. ${ }^{17}$ For the purposes of the current work, it is assumed that the difference between the samples from the two suppliers consists essentially of a different distribution of C-10 isomers that are not distinguishable by GC. In subsequent sections, it is shown that the samples from either of these two different sources, with a minimum purity of $99.8 \%$ (GC), seem to have appropriate chemical characteristics to become an industrial reference fluid. That is, even if there is a variation in chemical composition among the samples, its effect upon the viscosity seems to be within the uncertainty of measurement. These characteristics of DIDP are of importance when considering its suitability as a standard reference fluid in addition to some characteristics mentioned previously, ${ }^{20}$ such as the low vapor pressure, ${ }^{21}$ a relatively low toxicity ("no hazardous product according to EC Directive 67/548/EEC"22), and the availability of a large number of studies about its toxicity. The liquid phase of DIDP is stable at room temperature, under high pressures, ${ }^{16}$ which is a favorable asset for its eventual use as a viscosity standard at high pressures. However, although the liquid phase extends over a large range of temperatures, the utilization of DIDP for the present purposes should avoid heating above $60^{\circ} \mathrm{C}$ (see section 4.1). This impediment was determined by the findings of Fröba and Leipertz. ${ }^{23}$ Moreover, it should be pointed out that, according to the corresponding Merck KGaA Safety-Data-Sheet, heating should be avoided. ${ }^{22}$

\section{Thermophysical Properties of DIDP}

All the data used for the definition of the proposed standard were obtained using samples of DIDP with gas chromatography certified assays higher than $99.8 \%,{ }^{18,19}$ except one of the samples utilized for the discussion in section 3.1. The former samples have two distinct origins. All the data sets presented, except one (see Table 1), were obtained with samples from Merck KGaA, identified as diisodecyl phthalate GR for analysis according to DIN 75201 (Batch K22132622). The other data set was obtained with a sample from Sigma-Aldrich as diisodecyl phthalate, Fluka Selectophore (Lot 415288). In subsection 3.1.3, viscosity measurements obtained with the latter sample are compared with the results obtained, using the same instrumentation, with a sample from Merck KGaA with the same purity, according to their respective certificates of analysis. ${ }^{18,19}$

\subsection{Effect of Impurities on the Viscosity and the Density} of DIDP. 3.1.1. Effect of Water on the Viscosity. The effect of water contamination on the viscosity of DIDP has been reported by several authors. In the first of such studies, ${ }^{9}$ it has been stated that the viscosity of a sample of $99.8 \%$ purity from Merck KGaA containing a mass fraction of water of about $100 \cdot 10^{-6}$ was found to be $0.5 \%$ lower than the viscosity of an otherwise identical sample containing approximately a mass fraction of water of $20 \cdot 10^{-6}$. This difference is less than the estimated overall uncertainty of the measurements involved in the comparison but is commensurate with the reproducibility of the vibrating-wire measurements ${ }^{9,15,24}$ used in that study. The work carried out by Fröba and Leipert ${ }^{23}$ has confirmed the order of magnitude of the effect. Further confirmation has recently been produced by $\mathrm{Al}$ Motari et al. ${ }^{24}$ In particular, the latter authors have measured the viscosity of DIDP samples containing water mass fractions from $\left(20 \cdot 10^{-6}\right.$ to $\left.417 \cdot 10^{-6}\right)$, and concluded that the differences found were within the expanded uncertainty $( \pm 2 \%)$ of their measurements at atmospheric pressure. Therefore, the studies performed so far support the observation that a 5-fold increase in water content of the DIDP samples is responsible for a decrease in viscosity of the order of $0.5 \%{ }^{9}$ 


\subsubsection{Effect of Impurities Detected by GC on the}

Viscosity. The effect of other kinds of impurities on the viscosity of samples of DIDP has also been tackled. In particular, several researchers studied samples from Merck KGaA with purities of $99 \%$ and $99.8 \%$ to assess the effect of impurities on the viscosity of DIDP samples. The difference of viscosity between two such samples has been obtained at $293.15 \mathrm{~K}$, by Bauer, ${ }^{25}$ using capillary viscometry. The value obtained for the difference $(1.2 \%)$ has an extremely low estimated uncertainty, of the order of $\pm 0.05 \%$ of the viscosity at a $95 \%$ confidence level. ${ }^{25}$ The differences encountered by Caetano et al., ${ }^{15,26}$ Harris and Bair, ${ }^{16}$ and $\mathrm{Al}$ Motari et al., ${ }^{24}$ using methods yielding much larger uncertainties, are consistent with the above result. The conclusion is, therefore, that if differences in the purity of DIDP samples can be detected using gas chromatography then viscosity differences of the order of $\pm 1 \%$ are likely to be observed. This is of importance if a viscosity calibration accuracy of the same order is sought.

3.1.3. Effect of Impurities Not Detected by GC on the Viscosity. As mentioned above (see section 2, Characterization of the DIDP Samples Used in the Present Work), the samples of DIDP are isomeric mixtures, and in principle, it is to be expected that different production lines may yield different distributions of C-10 isomers in the respective final products. However, evidence exists that two high-purity samples originating from different producers have a viscosity that differs by less than the uncertainty of the measurements. This may be inferred from a comparison of the measurements performed by Caetano et al. on a Sigma-Aldrich DIDP Fluka, $99.8 \%$, sample ${ }^{26}$ and on a Merck KGaA, $99.8 \%$, sample. ${ }^{15}$ Both sets of measurements have been performed with a capillary viscometer, which has been previously calibrated by Schott-Instruments. The calibration constant value ${ }^{27}$ was, ex post, confirmed to differ less than $0.16 \%$ from the value obtained using a PTB standard liquid specimen 100B. The measurements of the viscosity of the Fluka sample were carried out at (293.15 and 298.15) K, yielding the values (123.8 and 88.83$) \mathrm{mPa} \cdot \mathrm{s}$, respectively. These results differ less than $\pm 0.3 \%$ from the viscosity of a Merck KGaA (99.8 \% GC) DIDP sample measured with the same viscometer. ${ }^{15}$

The estimated uncertainty of the measurements is $\pm 1.5 \%$, at a $95 \%$ confidence level, and the reproducibility of each measurement is better than $\pm 0.4 \%$. The viscosity differences found are well within the uncertainty of the method and commensurate with the reproducibility of the measurements. This indicates that the likely different isomeric composition between the two samples does not change the viscosity within the estimated reproducibility of the capillary measurements performed. Recently, Al Motari et al. ${ }^{24}$ have measured the viscosity of a Fluka (99.9 \% GC) sample and a Merck KGaA (99.8\% GC) sample at $298.15 \mathrm{~K}$. Those authors reported ${ }^{24}$ values that differ less than $\pm 0.35 \%$, which confirms the above conclusions.

3.1.4. Effect of Impurities on the Density. The effect of impurities, including the effect of small amounts of water, on the density of DIDP samples has been shown to be much smaller than on the viscosity. In particular, the differences in density found in the study referred to above ${ }^{9}$ between samples of DIDP containing water in excess of $100 \mathrm{mg} \cdot \mathrm{kg}^{-1}$ and about 20 $\mathrm{mg} \cdot \mathrm{kg}^{-1}$, respectively, were not greater than $0.02 \%$. Subsequently, several works have confirmed this lack of sensitivity of the density to impurities in DIDP samples. One such study has been reported by Al Motari et al., ${ }^{24}$ who have concluded that the differences in density at atmospheric pressure between samples with purities of $99 \%$ and $99.8 \%$ were not greater than the expanded uncertainty of their measurements $( \pm 0.1 \%)$, and the same was observed when they compared the density of DIDP samples with water mass fractions, $w$, in the range $20 \leq w \leq$ $417 \cdot 10^{-6}$. Moreover, Harris and Bair ${ }^{16}$ found that DIDP samples from Merck KGaA with purities of $99 \%$ and $99.8 \%$, respectively, had densities at atmospheric pressure that were essentially identical within the uncertainty of the experimental measurements, which was estimated by those authors to be \pm $0.00005 \mathrm{~g} \cdot \mathrm{cm}^{-3}$.

3.2. Density Data. Most of the experimental techniques used to measure the viscosity require a knowledge of the density, and therefore most of the authors who have measured the viscosity of DIDP have also measured the density of DIDP samples, with purity greater than $99.8 \%$ (GC), at atmospheric pressure. Table 1 summarizes the DIDP density data sets selected for the present proposal, including the experimental techniques used. All the data have uncertainties lower than \pm $0.1 \%$. Besides the data sets shown in Table 1, another data set of measurements of the density of DIDP that has been published ${ }^{24}$ has not been included for reasons expounded below.

Each of the 18 data points of the set in Table 1, obtained by Caetano et al., with refs 15 and 26, are, for each temperature, the arithmetic mean of five density measurements, shown as Supporting Information to this article. Also shown as Supporting Information are the unpublished data sets obtained by Fröba and Leipertz, ${ }^{30}$ Bauer, ${ }^{25}$ and Caetano et al. ${ }^{26}$ and the data obtained by Peleties and Trusler. ${ }^{28,29}$

The density measurements of DIDP summarized in Table 1, obtained in the range ( 281.15 to 315.15 ) $\mathrm{K}$, were fitted to a polynomial equation of the form

$\frac{\rho}{\mathrm{kg} \cdot \mathrm{m}^{-3}}=a+b(T / \mathrm{K}-273.15)+c(T / \mathrm{K}-273.15)^{2}+$

The objective function that was minimized for the fitting is ${ }^{31}$

$$
\sum_{i=1}^{N} w_{i}\left(\rho_{i}-\rho\left(T_{i}\right)\right)^{2}
$$

where $N$ is the number of experimental points; $\rho_{i}$ is the $i$ th experimental point of density; and $\rho\left(T_{i}\right)$ is the corresponding calculated value at temperature $T_{i}$. The statistical weights $w_{i}$ of the different density data sets were assumed equal.

The statistical information in Table 1, concerning each data set, consists of the relative root-mean-square deviation, rmsd, and the bias defined as follows

$$
\operatorname{rmsd}=\left[\frac{1}{N_{\mathrm{p}}} \sum_{i=1}^{N_{\mathrm{p}}}\left(\frac{\rho_{i}-\rho\left(T_{i}\right)}{\rho\left(T_{i}\right)}\right)^{2}\right]^{1 / 2}
$$

and

$$
\text { bias }=\frac{1}{N_{\mathrm{p}}} \sum_{i=1}^{N_{\mathrm{p}}}\left(\frac{\rho_{i}-\rho\left(T_{i}\right)}{\rho\left(T_{i}\right)}\right)
$$

where $N_{\mathrm{p}}$ is the number of points of the data set considered.

The parameters of the fitting of eq 1 are shown in Table 2, together with the rmsd and bias of all the points. This equation will be used to calculate the recommended density values of DIDP at the temperatures selected to define the viscosity standard values.

Figure 1 shows a deviation plot of the experimental density data from the polynomial eq 1 . It can be seen that the maximum 
Table 2. Fitting Parameters of Equation 1, Together with the Relative Root Mean Square Deviation (rmsd) and Bias of the Density Data from the Fitting

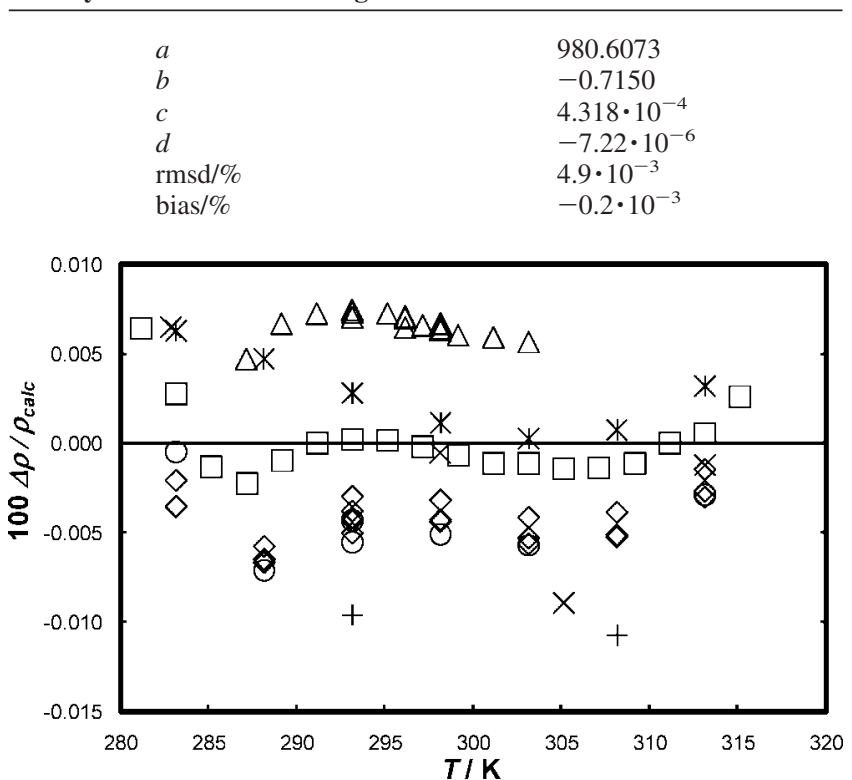

Figure 1. Relative deviations, $\Delta \rho=\rho_{\text {exptl }}-\rho_{\text {calcd }}$, of the atmospheric pressure density data, $\rho_{\text {expt }}$, of liquid DIDP, summarized in Table 1, from the values, $\rho_{\text {calcd }}$, obtained with the correlation eq 1 , with fitting parameters shown in Table 2. $\square$, Caetano et al. (DIDP sample from Merck KGaA) $;^{15}$ $\bigcirc$, Harris and Bair; ${ }^{16} \times$, Peleties and Trusler; ${ }^{28,29} \triangle$, Caetano et al. ${ }^{26}$ (DIDP sample from Fluka); +, Bauer; ${ }^{25} \diamond$, Fröba and Leipertz $(2005) ;{ }^{30} *$, Fröba and Leipertz. ${ }^{23}$

deviation does not exceed $0.011 \%$ which is less than the estimated relative uncertainty of the data sets.

Al Motari et al. ${ }^{24}$ published several measurements of the density of DIDP at $298.15 \mathrm{~K}$, within the temperature range (283 to 318 ) K. However, two of those values, obtained with two different vibrating-tube instruments, concerning the density of the same sample [sample B in ref 24, i.e., Merck KGaA (99.8 $\%$ by GC) with a water weight fraction of $417 \cdot 10^{-6}$ ] differ from each other by $0.39 \%$. It is also noteworthy that the measurement made by the most accurate of those instruments ${ }^{24}$ at $298.15 \mathrm{~K}$ differs from the present correlation of density data by $0.1 \%$, which is 1 order of magnitude larger than the average deviation of the remaining results to the fitting equation. For this reason, the density data published by those authors were not included for the definition of the density of the viscosity standard.

3.3. Viscosity Data. A significant number of measurements of the viscosity of DIDP have been performed recently. To the best knowledge of the authors, the first measurements described in the specialized literature were obtained with a preliminary version of a purpose built vibrating-wire instrument. ${ }^{20}$ Following this initial set of measurements, the apparatus and the experimental technique were developed further, ${ }^{32}$ to improve the overall uncertainty of the measurements and to expand the available range of viscosity. A new set of viscosity measurements of DIDP has been published ${ }^{17}$ by the same authors, which is directly traceable to the primary standard reference value-the viscosity of water ${ }^{2}$ at $293.15 \mathrm{~K}$-and have an overall uncertainty of $\pm 0.8 \%$, at temperatures in the vicinity of $293 \mathrm{~K}$ and higher, and an uncertainty that does not exceed $\pm 1 \%$, at lower temperatures.

Table 3 summarizes the viscosity measurements of DIDP, with purity greater than $99.8 \%$ (GC), obtained at atmospheric pressure, through a variety of experimental techniques that have been selected to define the present proposal for viscosity standard. The criteria used for this selection are expounded below.

The uncertainties, $u$, shown in the third column of Table 3 are the estimated relative uncertainties of the respective data sets, as claimed by their authors. In addition, it should be pointed out that viscosity measurements in the compressed liquid state have also been carried out recently, ${ }^{16,24,28,29}$ although not directly relevant to this study. The unpublished viscosity data points used to obtain the correlation, eq 5 , measured by Bauer ${ }^{25}$ and by Caetano et al. $^{26}$ are shown in Table 6 , and those obtained by Peleties and Trusler ${ }^{28,29}$ are available as Supporting Information of the present work. It should be noted that we have not included in Table 3 the results of Al Motari et al. ${ }^{24}$ for the viscosity of DIDP for reasons explained below. However, we note that the values determined by Al Motari et al. ${ }^{24}$ depart by significantly less than their estimated uncertainty $( \pm 2 \%)$ from the reference values we propose.

All the viscosity data, in the temperature range $288.15 \mathrm{~K} \leq$ $T \leq 308.15 \mathrm{~K}$, indicated in Table 3 , have been correlated with temperature by a Vogel-type equation ${ }^{33}$

$$
\eta /(\mathrm{mPa} \cdot \mathrm{s})=\exp \left(A+\frac{1000 B}{C+T}\right)
$$

The fitting parameters $A, B$, and $C$, are shown in Table 4 , together with statistical information concerning the data correlation, namely, the relative root-mean-square deviation, rmsd, and bias, as defined below. The objective function that has been minimized for the fitting is ${ }^{31}$

$$
\sum w_{i}\left(\eta_{i}-\eta\left(T_{i}\right)\right)^{2}
$$

where $N$ is the number of experimental points; $\eta_{i}$ is the $i$ th experimental point of viscosity; and $\eta\left(T_{i}\right)$ is the corresponding calculated value at temperature $T_{i}$. The statistical weights, $w_{i}$, of the different viscosity data sets were assumed equal.

The statistical information in Table 3 , concerning each of the different viscosity data sets, is the relative root-mean-square deviation, rmsd, and the bias defined as follows

$$
\operatorname{rmsd}=\left[\frac{1}{N_{\mathrm{p}}} \sum_{i=1}^{N_{\mathrm{p}}}\left(\frac{\eta_{i}-\eta\left(T_{i}\right)}{\eta\left(T_{i}\right)}\right)^{2}\right]^{1 / 2}
$$

and

$$
\text { bias }=\frac{1}{N_{\mathrm{p}}} \sum_{i=1}^{N_{\mathrm{p}}}\left(\frac{\eta_{i}-\eta\left(T_{i}\right)}{\eta\left(T_{i}\right)}\right)
$$

where $N_{\mathrm{p}}$ is the number of points of the data set considered.

Figure 2 shows a deviation plot of the experimental viscosity data from the correlation represented by eq 5 . It can be seen that the maximum deviation of the data sets, shown in Table 3, does not exceed $\pm 0.5 \%$, which is commensurate with, or smaller than, their estimated relative uncertainty.

A comparison with the data of Al Motari et al. ${ }^{24}$ at three temperatures and atmospheric pressure is also possible from Figure 2 . The data published by Al Motari et al. concern mainly measurements at pressures higher than $5 \mathrm{MPa}$. Only four data points were measured at $0.1 \mathrm{MPa}$ with samples of nominal purity greater than $99.8 \%$ (GC): one (at $T=298.15 \mathrm{~K}$ ) was obtained with a sample from Fluka (Sigma-Aldrich), and the other three (at $T=298.15 \mathrm{~K}, T=303.15 \mathrm{~K}$, and $T=308.15 \mathrm{~K}$ ) with a sample from Merck KGaA (we note that the densities at the two higher temperatures are not given in ref 24). Both points at $298.15 \mathrm{~K}$ show negative deviations exceeding $-1 \%$, which 
Table 3. Measurements of the Viscosity of DIDP with Purity Greater than $99.8 \%$ (GC), at Atmospheric Pressure, Included in the Formulation of the Standard Reference Value ${ }^{a, b}$

\begin{tabular}{|c|c|c|c|c|c|c|c|c|}
\hline method & origin \& purity & nominal uncertainty, $u / \%$ & $T / \mathrm{K}$ & $N_{\mathrm{p}}$ & $\mathrm{rmsd} / \%$ & bias $/ \%$ & authors & ref \\
\hline capillary & Merck KGaA, $99.8 \%$ & \pm 0.31 & 293.15 & 1 & 0.22 & -0.22 & Bauer & 25 \\
\hline vibrating wire & idem & $\pm 0.8^{c}$ & (283.15 to 313.15 ) & 5 & 0.23 & -0.18 & Caetano et al. & 15 \\
\hline falling body & idem & \pm 2 & (273.15 to 353.15$)$ & 5 & 0.23 & 0.05 & Harris and Bair & 16 \\
\hline capillary & idem & \pm 1.5 & ( 273.97 to 368.38 ) & 2 & 0.16 & -0.16 & Peleties and Trusler & 28,29 \\
\hline capillary & idem & \pm 1.5 & $293.15,298.15$ & 2 & 0.14 & 0.12 & Caetano et al. & 26 \\
\hline capillary & Fluka, $99.8 \%$ & \pm 1.5 & $293.15,298.15$ & 2 & 0.40 & 0.39 & Caetano et al. & 26 \\
\hline
\end{tabular}

${ }^{a}$ The number of points, $N_{\mathrm{p}}$, is the number of experimental points in the temperature range (288.15 to 308.15$) \mathrm{K}$, used in the correlation eq 5 . The values for the relative root mean square deviation (rmsd) and Bias relate to the correlation eq $5 .{ }^{b}$ Note: The unpublished viscosity data points used to obtain the correlation eq 5 , measured by Bauer $^{25}$ and by Caetano et al., ${ }^{26}$ and the data points obtained by Peleties and Trusler ${ }^{28,29}$ are available as Supporting Information. ${ }^{c} \pm 1.0 \%$ for $\eta \geq 130 \mathrm{mPa} \cdot \mathrm{s}$.

Table 4. Parameters of Equation 5 that Fit the Experimental Viscosity Measurements and Corresponding Relative Root Mean Square Deviation (rmsd) and Bias of the Viscosity Data from the Fitting

$\begin{array}{ll}A /(\mathrm{mPa} \cdot \mathrm{s}) & -3.1736 \\ B / \mathrm{K} & 0.9151 \\ C / \mathrm{K} & -178.606 \\ \mathrm{rmsd} / \% & 0.23 \\ \mathrm{bias} / \% & -0.01\end{array}$

Table 5. Fitting Parameters for Equation 9, Together with the Relative Root Mean Square Deviation (rmsd) and Bias of the Surface Tension Data

\begin{tabular}{cccccc}
\hline author & $\gamma_{0} /\left(\mathrm{mN} \cdot \mathrm{m}^{-1}\right)$ & $n$ & $\mathrm{rmsd} / \%$ & bias $/ \%$ & ref \\
\hline Caetano et al. & 50.79 & 0.905 & 0.13 & 0.0 & 9
\end{tabular}

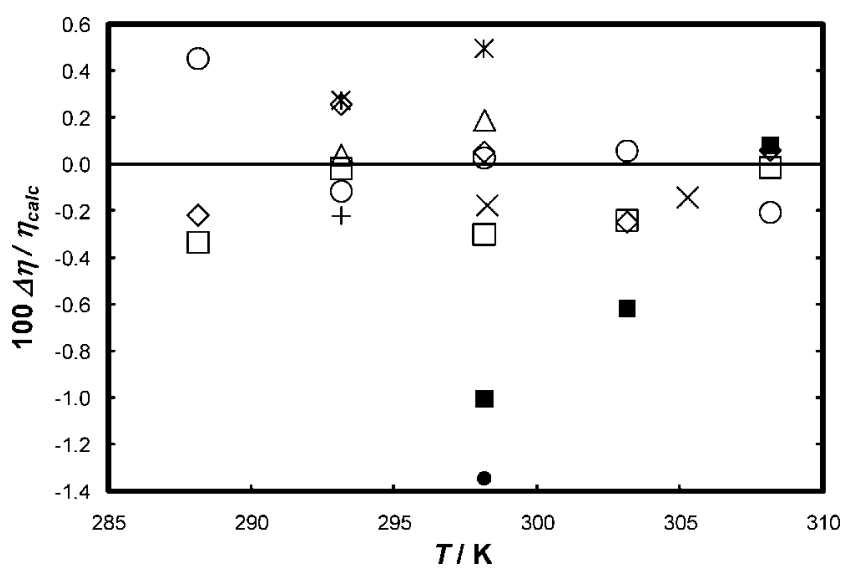

Figure 2. Relative deviations, $\Delta \eta=\eta_{\text {exptl }}-\eta_{\text {calcd }}$, of the atmospheric pressure viscosity data, $\eta_{\text {exptt }}$, of liquid DIDP, from the values, $\eta_{\text {calcd }}$, obtained with the correlation eq 5, with fitting parameters shown in Table 4. $\diamond$, Fröba and Leipertz; ${ }^{23} \square$, Caetano et al. ${ }^{15}$ (vibrating wire; DIDP sample from Merck KGaA); $\bigcirc$, Harris and Bair; ${ }^{16} \times$, Peleties and Trusler; ${ }^{28,29} *$, Caetano et al. ${ }^{26}$ (capillary; DIDP sample from Fluka); +, Bauer; ${ }^{25} \triangle$, Caetano et al. ${ }^{15,26}$ (capillary; DIDP sample from Merck KGaA); $\bullet$, Al Motari et al. ${ }^{24}$ (DIDP sample from Fluka); $\mathbf{\square}$, Al Motari et al. ${ }^{24}$ (DIDP sample from Merck KGaA).

is larger than twice the rmsd of the remaining data from the correlation of eq 5 . In fact, only the point at the lowest viscosity (at $T=308.15 \mathrm{~K}$ ) has a deviation lying within an interval of twice the rmsd of the correlation of eq 5 . Thus, as the data points of Al Motari et al. ${ }^{24}$ differ significantly from the consensus of the other data, they were excluded from the set of measurements used for the definition of the standard. However, as remarked before, those same data deviate by less than their estimated uncertainty from the correlation of eq 5 , which certainly enables their use in exploring the effect of water upon the viscosity of DIDP.

3.4. Surface Tension Data. Only one article could be found in the literature describing measurements of the surface tension, $\gamma$, of DIDP. ${ }^{9}$ The experimental method used is based on the analysis of the shape of a pendant drop, and the results have a nominal relative uncertainty of $\pm 0.5 \%$. The measurements ${ }^{9}$ extended over the temperature range $(288.15$ to 308.15$) \mathrm{K}$. Those results were fitted to an equation of the type

$$
\gamma=\gamma_{0}\left(1-T_{\mathrm{r}}\right)^{n}
$$

where $\gamma_{0}$ and $n$ are constant parameters and $T_{\mathrm{r}}=T / T_{\mathrm{c}}$ is the reduced temperature. The critical temperature of DIDP has been estimated using the method of Marrero-Marejón and PardilloFontdevila, ${ }^{34}$ as described in ref 35 , the result being $T_{\mathrm{c}}=670$ $\mathrm{K}$. This kind of equation is reported by Poling et al. ${ }^{35}$ to describe the temperature variation of surface tension adequately and was selected for interpolation purposes in the modest temperature range of the available results for DIDP. The objective function and the statistical analysis were of the same type as described above for viscosity and density. The fitting parameters, as well as the rmsd and the bias, are shown in Table 5.

Equation 9 will be used to establish the value of the surface tension of the DIDP reference sample at the selected temperatures.

\section{Industrial Reference Standard}

In this section, we use the results set out in the previous section to define a standard reference sample of DIDP for industrial use as a reference material for viscosity of moderate viscosity. We set out how the sample of DIDP is to be prepared and subsequently define the viscosity, density, and surface tension to be used in the calibration of a viscometer.

4.1. Sample Preparation. The proposed reference fluid is diisodecyl phthalate (DIDP) with a purity in excess of $99.8 \%$ as determined using gas chromatography by the suppliers. Although the effect of the presence of small amounts of water in the DIDP samples may have a limited influence on their viscosity, previous experience ${ }^{9,15,23}$ has suggested that the samples of DIDP $(99.8 \%)$ should receive a treatment prior to their use as a reference fluid that consists of the removal of water by means of $0.4 \mathrm{~nm}$ molecular sieves. For this purpose, standard procedures should be followed. Samples treated with activated molecular sieves for at least $24 \mathrm{~h}$, with occasionally repeated manual stirring, have reached water mass fractions of $20 \cdot 10^{-6}$, as measured by coulometric Karl Fischer titration. ${ }^{15}$ Mechanical agitation should be avoided as that can promote the degradation of the molecular sieve pellets. Subsequent filtration of the samples prior to use through a (40 to 60) $\mu \mathrm{m}$ filter is also recommended.

Degassing of the samples is also advisable, to avoid bubble formation, especially in capillary measurements. For this purpose, it is recommended to use sparging with helium. Degassing under a vacuum and mild heating is also possible; however, it is not recommended to submit the sample to 
Table 6. Viscosity Data, $\eta /(\mathrm{mPa} \cdot \mathrm{s})$, Used to Establish the Reference Values for Viscosity ${ }^{a}$

\begin{tabular}{|c|c|c|c|c|c|c|}
\hline author & ref & method & uncertainty, $u / \%$ & $T / \mathrm{K}=293.15$ & $T / \mathrm{K}=298.15$ & $T / \mathrm{K}=303.15$ \\
\hline Bauer & 25 & capillary & \pm 0.31 & 123.19 & - & - \\
\hline Caetano et al. & 15 & vibrating wire & \pm 0.8 & 123.44 & 88.30 & 65.06 \\
\hline Harris and Bair & 16 & falling body & \pm 2 & 123.32 & 88.42 & 65.04 \\
\hline Fröba and Leipertz & 23 & SLS & \pm 1.4 & 123.78 & 88.44 & 64.84 \\
\hline Peleties and Trusler & 28,29 & capillary & \pm 1.5 & - & 88.31 & - \\
\hline Caetano et al. & 26 & capillary & \pm 1.5 & 123.51 & 88.56 & - \\
\hline $\begin{array}{l}\text { Caetano et al. } \\
\text { (sample from Fluka) }\end{array}$ & 26 & capillary & \pm 1.5 & 123.80 & 88.83 & - \\
\hline
\end{tabular}

${ }^{a}$ The nominal uncertainties were given by the respective authors.

Table 7. Reference Values for the Viscosity, $\eta_{\text {ref }}$, of DIDP at 293.15 $\mathrm{K}, 298.15 \mathrm{~K}$, and 303.15 K, and Their Respective Repeatability, $r_{\eta}{ }^{a}$

\begin{tabular}{|c|c|c|c|c|}
\hline$T / \mathrm{K}$ & $\eta_{\mathrm{ref}} / \mathrm{mPa} \cdot \mathrm{s}$ & $r_{\eta} / \mathrm{mPa} \cdot \mathrm{s}$ & $\rho / \mathrm{kg} \cdot \mathrm{m}^{-3}$ & $\gamma / \mathrm{mN} \cdot \mathrm{m}^{-1}$ \\
\hline 293.15 & 123.5 & 0.5 & 966.42 & 30.17 \\
\hline 298.15 & 88.5 & 0.4 & 962.89 & 29.80 \\
\hline 303.15 & 65.0 & 0.3 & 959.35 & 29.44 \\
\hline
\end{tabular}

${ }^{a}$ Density and surface tension values are also given for the same temperatures. Viscosity and density values were obtained as described in the text, from the references shown in Tables 1 and 3, respectively. The surface tension was obtained as described in the text using source data from ref 9 .

temperatures higher than $60{ }^{\circ} \mathrm{C}$ at any time. This explicit recommendation is made on the basis of studies conducted by Fröba and Leipertz ${ }^{23}$ who have observed modifications of the properties of samples submitted to temperatures higher than 100 ${ }^{\circ} \mathrm{C}$.

4.2. Reference Data. Reference values for the viscosity of DIDP at atmospheric pressure have been determined at (293.15, 298.15 , and 303.15) K. The original data used to obtain the reference values are listed in Table 6 , together with their original sources. The values obtained by Caetano et al., using the vibrating wire technique, shown in Table 6 , are the arithmetic mean of the values reported by those authors ${ }^{15}$ after correction to the above reference temperatures, over very small temperature intervals (e.g., (293.21 to 293.15) K), using their data correlation equation. Similarly, the datum ${ }^{28,29}$ from Peleties and Trusler at 298.25 K was corrected to $298.15 \mathrm{~K}$, using eq 5 . The values shown from Harris and Bair ${ }^{16}$ are the arithmetic mean of the respective values reported by those authors at the corresponding reference temperatures.

The reference data are presented in Table 7. The reference viscosity values, $\eta_{\text {ref }}$, for each temperature have been calculated as the arithmetic mean of the available individual source values, $\eta_{i}$, in Table 6 . For each temperature, the repeatability of the reference value, $r_{\eta}$, in Table 7 is estimated as twice the standard deviation, $\sigma$, of the individual values in Table 6 , from the mean

$$
\sigma=\left[\frac{\sum\left(\eta_{i}-\eta_{\mathrm{ref}}\left(T_{i}\right)\right)^{2}}{N_{\mathrm{p}}-1}\right]^{1 / 2}
$$

where $N_{\mathrm{p}}$ is the number of experimental points of Table 6 used to calculate the reference value $\eta_{\mathrm{ref}}\left(T_{i}\right)$ at temperature $T_{i}$, and $\eta_{i}$ is the $i$ th viscosity value in Table 6 .

Figure 3 shows a deviation plot of the data used to calculate the three mean values for the viscosity, shown in Table 7 . The overall uncertainties of the reference viscosity data, considering the existence of systematic uncertainties affecting the different data sets in Table 6, in addition to random effects, are estimated to be of the order of $\pm 1 \%$.

The values shown in Table 9 for the density and the surface tension have been obtained by means of eqs 1 and 9. The repeatability of the corresponding values in Table 7, at a $95 \%$

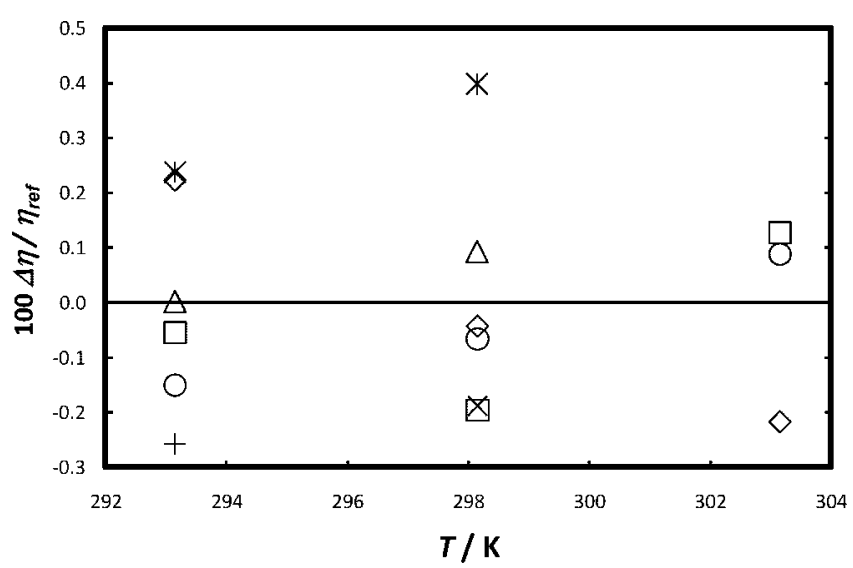

Figure 3. Relative deviations, $\Delta \eta=\eta_{\text {exptl }}-\eta_{\text {ref }}$, of the experimental viscosity values, $\eta_{\text {expt }}$, in Table 6 , from the proposed reference values, $\eta_{\text {ref }}$, for DIDP, shown in Table 7. $\diamond$, Froba and Leipertz; ${ }^{23} \square$, Caetano et al. ${ }^{15}$ (vibrating wire; DIDP sample from Merck KGaA); $\bigcirc$, Harris and Bair; ${ }^{16}$ $\times$, Peleties and Trusler; ${ }^{28,29} *$, Caetano et al. ${ }^{26}$ (capillary; DIDP sample from Fluka); + , Bauer; ${ }^{25} \triangle$, Caetano et al. ${ }^{15,26}$ (capillary; DIDP sample from Merck $\mathrm{KGaA}$ ).

confidence level, is estimated to be smaller than $0.1 \%$ for the density and $1 \%$ for the surface tension.

For performing small corrections to calculate the viscosity of DIDP in the vicinity of the reference temperatures shown in Table 7, eq 5 should be used with the fitting parameters given in Table 4. For this purpose, given a temperature $T$, in the vicinity of one of the reference temperatures shown in Table 7 , $T_{\text {ref }}$, the viscosity, $\eta(T)$, at temperature $T$ is given by

$$
\begin{aligned}
\eta(T) /(\mathrm{mPa} \cdot \mathrm{s})=[ & \left.\eta\left(T_{\text {ref }}\right) /(\mathrm{mPa} \cdot \mathrm{s})\right] \exp \cdot \\
& {\left[1000 B\left(\frac{T_{\text {ref }}-T}{C^{2}+C\left(T_{\text {ref }}+T\right)+T_{\text {ref }} T}\right)\right] }
\end{aligned}
$$

where $B$ and $C$ have the same values as for eq 5 and are given in Table 4.

\section{Conclusions}

The paper proposes the adoption of DIDP as a reference fluid for viscosity for industrial purposes. The new reference fluid has a viscosity of $(123.5 \pm 0.5) \mathrm{mPa} \cdot \mathrm{s}$ at $293.15 \mathrm{~K}$, at atmospheric pressure. The interval of $\pm 0.5 \mathrm{mPa} \cdot \mathrm{s}$ corresponds to the repeatability of the reference value, estimated as twice the standard deviation from the mean, as defined by eq 10 .

To be used as an industrial reference fluid, DIDP should have a minimum purity of $99.8 \%$ as determined by GC. A pretreatment aimed at the reduction of the water content and degassing before use are recommended. Reference values for the viscosity and complementary data for the density and surface 
tension have been given at three temperatures to enable the selection of the most convenient calibration procedure for each application. The relative uncertainties of the viscosity reference values are estimated to be of the order of $\pm 1 \%$.

It is noteworthy that measurements of the viscosity of DIDP at high pressure are now available in the literature, ${ }^{16,24}$ which may contribute to eventually widen the use of DIDP as a reference fluid in the future.

\section{Acknowledgment}

This work has been conducted under the guidance of the International Association for Transport Properties (IATP-http://transp.cheng. auth.gr/iatp.html). The authors thank Dr. Harro Bauer, PhysikalischTechnische Bundesanstalt (PTB), Braunschweig, Germany, for valuable discussions, and particularly for communicating unpublished data on the viscosity and density of DIDP. The authors also thank Dr. F. Peleties, Imperial College, U. K., for communicating data on DIDP before publication. Special thanks are also due to Professor M. Marques, of Centro de Química Estrutural, Instituto Superior Técnico, Lisbon, Portugal, for the interpretation of the ${ }^{13} \mathrm{C}$ NMR and GC-MS analyses performed in Lisbon. These thanks are extended to Professor José Ascenso and Dr. Conceição Oliveira, of the same institution, for their assistance with the ${ }^{13} \mathrm{C} \mathrm{NMR}$ and GC-MS analyses, respectively. KRH acknowledges the assistance of Dr. Lawrie Woolf with density measurements. The authors also thank Professor K. N. Marsh, for his valuable advice and suggestions for improvement of the manuscript.

\section{Supporting Information Available:}

Tables A1-A6. This material is available free of charge via the Internet at http://pubs.acs.org.

\section{Literature Cited}

(1) Marsh, K. N. Role of reference materials for the realization of physicochemical properties. Past, present, and future. Pure Appl. Chem. 2000, 72, 1809-1818.

(2) ISO/TR 3666-1998; Viscosity of water; 1998.

(3) Wakeham, W. A.; Nagashima, A.; Sengers, J. V. Reference Data, In Measurement of the Transport Properties of Fluids; Experimental Thermodynamics; Wakeham, W. A., Nagashima, A., Sengers, J. V., Eds.; Blackwell Scientific Publications: Oxford, U.K., 1991; Vol. 3, pp 439-451.

(4) Künzel, W.; Van Wijk, H. F.; Marsh, K. N. Viscosity. In Recommended Reference Materials for the Realization of Physicochemical Properties; Marsh, K. N., Ed.; (IUPAC), Blackwells Scientific Publications: Cambridge, MA, 1987; pp 45-72.

(5) Lundstrom, R.; Goodwin, A. R. H.; Hsu, K.; Frels, M.; Caudwell, D. R.; Trusler, J. P. M.; Marsh, K. N. Measurement of the Viscosity and Density of Two Reference Fluids, with Nominal Viscosities at $T$ $=298 \mathrm{~K}$ and $p=0.1 \mathrm{MPa}$ of (16 and 29) $\mathrm{mPa} \cdot \mathrm{s}$, at Temperatures between (298 and 393) K and Pressures below 55 MPa. J. Chem. Eng. Data 2005, 50 (1787), 1377-1388.

(6) Daborn, J E. The Viscosity Scale of the NPL. Metrologia 1975, 11, $79-84$.

(7) Goncalves, F. A.; Kestin, J.; Sengers, J. V. Surface-Tension Effects in Suspended-Level Capillary Viscometers. Int. J. Thermophys. 1991, $12,1013-1028$.

(8) Bauer, H.; Meerlender, G. Precise Viscosity Measurements of Newtonian Liquids with $\mu$ less-than $1 \mathrm{~mm}^{2} / \mathrm{s}$ for the Selection of Suitable Standards. Rheol. Acta 1984, 23, 514-521.

(9) Caetano, F. J. P.; Fareleira, J. M. N. A.; Fernandes, A.; Oliveira, C. M. B. P.; Serro, A. P.; Simões de Almeida, I. M.; Wakeham, W. A. Diisodecylphthalate (DIDP) - a potential standard of moderate viscosity: surface tension measurements and water content effect on viscosity measurements. Fluid Phase Equilib. 2006, 245, 1-5.

(10) Kawata, M.; Kurase, K.; Nagashima, A.; Yoshida, K. Capillary Viscometers. In Measurement of the Transport Properties of Fluids; Wakeham, W. A., Nagashima, A., Sengers, J. V., Eds.;Experimental Thermodynamics; Blackwell Scientific Publications: Oxford, U.K., 1991; Vol. 13, pp 49-75.

(11) European Chemicals Bureau - Institute for Health and Consumer Protection European Union Risk Assessment Report - 1,2-Benzene- dicarboxylic acid, di-C9-11-branched alkyl esters, $C$-10 rich and di"isodecyl" phthalate; EUR 20785 EN; PL-2, 36; European Commission, Joint Research Centre, 2003.

(12) Encyclopedia of Polymer Science and Technology, 3rd ed.; John Wiley \& Sons, Inc.: New York, 2003; Vol. 3, pp 498-524.

(13) Earls, A. O.; Axford, I. P.; Braybrook, J. H. Gas chromatographymass spectrometry determination of the migration of phthalate plasticisers from polyvinyl chloride toys and childcare articles. J. Chromatogr. A 2003, 983, 237-246.

(14) Gomez-Hens, A.; Aguilar-Caballos, M. P. Social and economic interest in the control of phthalic acid esters. Trends Anal. Chem. 2003, 22, $847-857$.

(15) Caetano, F. J. P.; Fareleira, J. M. N. A.; Oliveira, C. M. B. P.; Wakeham, W. A. New Measurements of the Viscosity of Diisodecyl Phthalate Using aVibrating Wire Technique. J. Chem. Eng. Data 2005, 50, 1875-1878.

(16) Harris, K. R.; Bair, S. Temperature and Pressure Dependence of the Viscosity of Diisodecyl Phthalate at Temperatures between (0 and 100) ${ }^{\circ} \mathrm{C}$ and at Pressures to $1 \mathrm{GPa}$. J. Chem. Eng. Data 2007, 52, 272278 .

(17) Marques, M. Centro de Química Estrutural; Instituto SuperiorTécnico, Universidade Técnica de Lisboa: Portugal, private communication, 2007.

(18) Certificate of Analysis, Merck KGaA, 1.03622.100 Diisodecyl Phthalate GR acc. DIN 75201, Batch K22132622; test date 28.07. 1995; test date 17.11.2004. http://www.merck-chemicals.com/.

(19) Sigma-Aldrich (Fluka), Certificate of Analysis, Diisodecyl Phthalate, Selectophore, Nov/04.

(20) Caetano, F. J. P.; Fareleira, J. M. N. A.; Oliveira, C. M. B. P.; Wakeham, W. A. Viscosity of Di-isodecylphthalate: A Potential Standard of Moderate Viscosity. Int. J. Thermophys. 2004, 25, 13111322.

(21) Merck Technical Data Sheet, 103622 diisodecyl phthalate GR for analysis acc. to DIN 75201, 1999.

(22) Safety Data Sheet, According to EC Directive 91/155/EEC, Merck $\mathrm{KGaA}$, date of issue: 14-10-2003.

(23) Fröba, A. P.; Leipertz, A. Viscosity of Diisodecyl Phthalate by Surface Light Scattering (SLS). J. Chem. Eng. Data 2007, 52, 1803-1810.

(24) Al Motari, M. M.; Kandil, M. E.; Marsh, K. N.; Goodwin, A. R. H. Density and Viscosity of diisodecyl phthalate $\mathrm{C}_{6} \mathrm{H}_{4}\left(\mathrm{COOC}_{10} \mathrm{H}_{21}\right)_{2}$, with Nominal Viscosity at $T=298 \mathrm{~K}$ and $p=0.1 \mathrm{MPa}$ of $87 \mathrm{mPa} \cdot \mathrm{s}$, at Temperatures from (298.15 to 423.15$) \mathrm{K}$ and Pressures up to 70 MPa. J. Chem. Eng. Data 2007, 52, 1233-1239.

(25) Bauer, H. Physikalisch-Technische Bundesanstalt (PTB); Braunschweig: Germany, private communication, 2003.

(26) Caetano, F. J. P.; Fareleira, J. M. N. A.; Oliveira, C. M. B. P.; Wakeham, W. A., unpublished data, made available as Supporting Information to this article.

(27) Schott-Instruments GmbH, Germany, Manufacturer's Certificate for capillary viscometer (according to DIN 55350, part 18), apparatus $n^{\circ}$ 1012949, 2002.

(28) Peleties, F.; Trusler, J. P. M., data communicated prior to publication and made available as Supporting Information to this article.

(29) Peleties, F. Advanced Fluid Property Measurement for Oilfield Applications; PhD Thesis, University of London, 2008.

(30) Fröba, A. P.; Leipertz, A. unpublished data, made available as Supporting Information to this article.

(31) Bevington, P. R.; Keith Robinson, D. Data Reduction and Error Analysis for the Physical Sciences, 2nd ed.; McGraw-Hill Inc.: New York, 1992.

(32) Caetano, F. J. P.; Fareleira, J. M. N. A.; Oliveira, C. M. B. P.; Wakeham, W. A. Validation of a Vibrating-Wire Viscometer: Measurements in the Range of 0.5 to $135 \mathrm{mPa} \cdot$ s. J. Chem. Eng. Data 2005, 50, 201-205.

(33) Poling, B. E.; Prauznitz, J. M.; O'Connell, J. P. The Properties of Gases and Liquids, 5th ed.; McGraw-Hill: New York, 2001. Original references: (a) Vogel, H. Das Temperaturabhängigkeitgesetz der Viskosität von Flüssigkeiten (The temperature dependence of the viscosity of liquids). Phys. Z. 1921, 22, 645-646. (b) Fulcher, G. S. Analysis of recent measurements of the viscosity of glasses. J. Am. Ceram. Soc. 1925, 8, 339-355. (c) Tammann, G.; Hesse, W. Die Abhängigkeit der Viskosität von der Temperatur bei unterkühlten flüssigkeiten (The dependence of the viscosity on temperature for super-cooled liquids). Z. Anorg. Allg. Chem. 1926, 156, 245-257.

(34) Marrero-Marejón, J.; Pardillo-Fontdevila, E. Estimation of pure compound properties using group interaction contributions. AIChE J. 1999, 45, 615. as described in ref 35 .

(35) Poling, B. E.; Prauznitz, J. M.; O'Connell, J. P. The Properties of Gases and Liquids, 5th ed.; McGraw-Hill: New York, 2001.

Received for review January 24, 2008. Accepted May 28, 2008. Some of the authors (JMNAF, CMPBO, and FJPC) acknowledge support for 
their participation in the present project from Fundação para a Ciência e a Tecnologia (Portugal), with European Union funds (FEDER) and National funds (Portugal), under Project POCTI/EQU 34951/00. KRH acknowledges financial support from UNSW and from Schlumberger Technology Centre, Texas.

JE800059N 